Neurosurg Focus 8 (3):Article 5, 2000, Click here to return to Table of Contents

This article contains a video clip that can be viewed with QuickTime Player. Please click here to download QuickTime oftware.

\title{
The resolution of syringohydromyelia without hindbrain herniation after posterior fossa decompression
}

\author{
Bermans J. Iskandar, M.D., Gary L. Hedlund, D.O., Paul A. Grabb, M.D., \\ AND W. JERRY OAKES, M.D. \\ Division of Pediatric Neurosurgery and Department of Pediatric Imaging, Children's Hospital, \\ Birmingham, Alabama
}

\begin{abstract}
Object. To provide more information about this rare condition, the authors describe five cases of syringohydromyelia without hindbrain herniation. Preoperative magnetic resonance imaging with and without gadolinium-diethylenetriamine pentaacetic acid revealed no evidence of spinal cord tumor, arachnoiditis, or spinal dysraphism.

Methods. Craniocervical decompression was performed in all patients, after which there was resolution of the symptoms in the four symptomatic patients, and all five showed marked reduction in the size of the syrinx.

Conclusions. The authors hypothesize the rare occurrence of syringohydromyelia resulting from a Chiari-like pathophysiological condition but lacking a hindbrain hernia. Patients with this condition may benefit from craniocervical decompression.
\end{abstract}

\section{KEY WORDS • Chiari malformation surgery $\bullet$ hindbrain herniation}

In the 1890s, Professor Hans Chiari described four cerebellar anomalies, which were later termed the Chiari malformations. ${ }^{5,6}$ The Chiari malformations Types I, II, and III represent different degrees of herniation of posterior fossa tissue into the cervical canal (Chiari I and II), or through an upper cervical spina bifida (Chiari III), whereas the Chiari IV anomaly consists of hypoplasia of the cerebellum. Patients with Chiari I malformations may remain asymptomatic, have mild occipital headaches, or present with symptoms and signs of brainstem, upper spinal cord, and/or cerebellar dysfunction. One of the most common as well as one of the most ominous anomalies associated with these malformations is syringohydromyelia. In this report, we present five cases of syringohydromyelia without a defined hindbrain herniation, all of which improved with posterior fossa decompression. There was clinical improvement as well as a marked reduction in the size of the syrinx in all cases. This dramatic response to decompression indicates that this entity has a Chiari-like pathophysiology. The clinical, radiographic, and surgical findings in the five patients are presented, along with a brief discussion of the possible pathogenesis of the lesion.

\section{CLINICAL MATERIAL AND METHODS}

This study is a retrospective review of five patients with syringohydromyelia that lacked a hindbrain herniation, all of whom were treated with craniocervical decompression.

\section{Preoperative Work-Up}

Complete craniospinal imaging was performed preoperatively to rule out other causes of syringohydromyelia. Magnetic resonance (MR) images of the entire spine were obtained to rule out occult spinal dysraphism. All MR imaging was performed with and without gadolinium-diethylenetriamine pentaacetic acid to evaluate the possibility of spinal cord tumor or arachnoiditis as a cause of syringohydromyelia. In addition, all patients underwent cranial imaging (computerized tomography [CT] or MR imaging) to identify hydrocephalus or other associated brain anomalies. The presence of ventral craniocervical anomalies was also investigated on MR imaging. The technique of cine two-dimensional (2-D) phase-contrast flow analysis (cine MR imaging) was used to assess flow around the foramen magnum in the four patients most recently treated.

\section{Surgical Procedure}

All patients underwent craniocervical decompression as previously described. ${ }^{11}$ This consisted of a suboccipital craniectomy, C-1 laminectomy, duraplasty, and the assurance of good flow from the foramen of Magendie; this often necessitated an intradural lysis of adhesions and opening of a fourth ventricular veil. On occasion, we reduced one of the cerebellar tonsils to further expand the outlet foramen. 


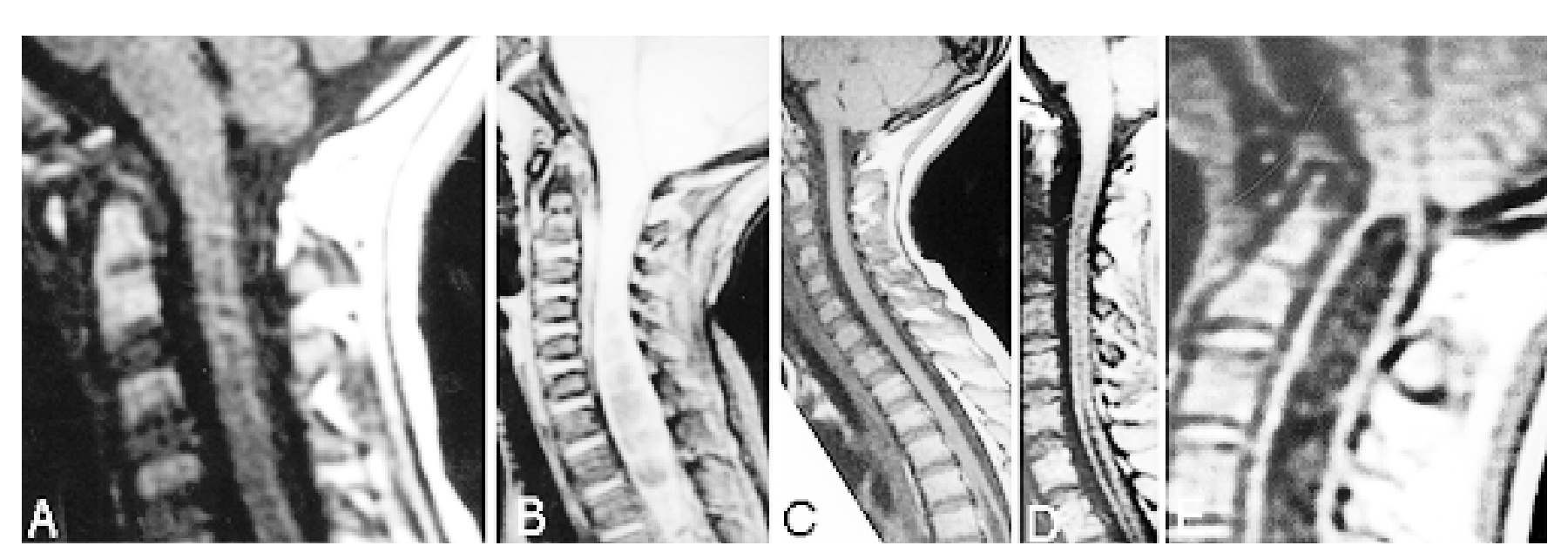

Fig. 1. Sagittal MR images of the craniocervical junction and cervical spine in Cases 1 to 5 (A-E), respectively. Note the lack of hindbrain herniation.

\section{Follow-Up Review}

The patients were followed closely to check for symptomatic and neurological improvement. Postoperative MR images were obtained to assess the size of the syrinx in all five patients, and postoperative cine MR imaging was performed in four of them.

\section{Estimation of Syrinx Size}

Although the syrinx volume is the most precise estimate of its size, the irregularity of this structure made it difficult to obtain reliable measurements. Therefore, the ratio of the surface area of syrinx to spinal cord was measured using the formula $\pi r^{2}$ for surface area, with $r$ being the radius. In addition, the diameter ratio was obtained at the level of maximum expansion to provide a simpler method of measurement. Pre- and postoperative ratios were compared at corresponding anatomical locations.

\section{Magnetic Resonance Imaging Techniques}

The MR images were obtained by using a 1.5-tesla scanner (General Electric Medical Systems, Milwaukee, WI). Our standard MR protocol consisted of spin-echo $\mathrm{T}_{1}$-weighted sequences in sagittal and axial orientations and fast spin-echo images in the sagittal plane. To supplement this protocol all patients received intravenous gadopentetate dimeglumine $(0.5 \mathrm{mmol} / \mathrm{kg}$ body weight $)$ to rule out occult tumor as a cause of syringohydromyelia. The craniocaudal extent, morphological appearance, width, and anteroposterior dimension of the syrinx were recorded.

\section{Cine MR Imaging (Video clips Normal or Abnormal}

In addition to standard spin-echo techniques, we performed cerebrospinal fluid (CSF) flow dynamic studies with the 2-D phase-contrast cine MR technique, which uses the motion-sensitive nature of MR imaging to demonstrate flow dynamics at the craniocervical junction. Normal CSF movement is relatively synchronous with arterial pulsations. ${ }^{9,13}$ We performed qualitative analysis of flow pre- and postoperatively. Lack of flow was seen as an intermediate shade of gray, cranial-to-caudal flow as high signal intensity (white) and caudocranial flow as low signal intensity (black). ${ }^{7}$

\section{RESULTS}

\section{Clinical and Radiographically Confirmed Improvement}

Five consecutive patients (two boys and three girls) with syringohydromyelia and no hindbrain herniation (Fig. 1) were followed with clinical and radiographic studies for a mean of 13.6 months and a range of 2 weeks to 36 months (Table 1). The age at operation ranged from 4 to 15 years, with a mean of 10.6 years. All five patients showed significant improvement in symptoms, signs, and syrinx size postoperatively, as detailed in Table 2 and Fig. 2. Two (Cases 1 and 2) of the three patients (Cases 1, 2,

TABLE 1

Summary of clinical and surgical findings in five patients with syringohydromyelia

\begin{tabular}{|c|c|c|c|c|c|c|}
\hline $\begin{array}{l}\text { Case } \\
\text { No. }\end{array}$ & $\begin{array}{l}\text { Length of } \\
\text { Follow Up }\end{array}$ & Bone & $\begin{array}{l}\text { Veil/Ad- } \\
\text { hesions }\end{array}$ & Symptoms & Signs & $\begin{array}{l}\text { Kypho- } \\
\text { scoliosis }\end{array}$ \\
\hline 1 & $8 \mathrm{mos}$ & C1-2 spina bifida & no & none & hyperreflexia; Babinski & no \\
\hline 2 & $19 \mathrm{mos}$ & normal & yes & leg pain & none & yes \\
\hline 3 & $3 \operatorname{mos}$ & normal & adhesions & back pain; leg paresthesias & none & no \\
\hline 4 & $2 \mathrm{wks}$ & normal & & interscapular pain & scoliosis & yes \\
\hline 5 & $36 \mathrm{mos}$ & assimilated $\mathrm{C}-1$ & adhesions & hand weakness & hand weakness & yes \\
\hline
\end{tabular}


Resolution of syringohydromyelia after posterior fossa decompression

TABLE 2

Summary of the imaging findings pre- and postoperatively*

\begin{tabular}{|c|c|c|c|c|c|c|c|c|}
\hline $\begin{array}{l}\text { Case } \\
\text { No. }\end{array}$ & Preop Flow & Postop Flow & $\begin{array}{c}\text { Preop Di- } \\
\text { ameter }(\mathrm{mm})\end{array}$ & $\begin{array}{c}\text { Epi- } \\
\text { center }\end{array}$ & Syrinx & $\begin{array}{c}\text { Preop Surface } \\
\text { Area }(\mathrm{mm})\end{array}$ & $\begin{array}{l}\text { Postop Di- } \\
\text { ameter (mm) }\end{array}$ & $\begin{array}{c}\text { Postop Surface } \\
\text { Area }(\mathrm{mm})\end{array}$ \\
\hline 1 & normal/decreased & improved & $0.41(7: 17)$ & $\mathrm{T}-8$ & $\mathrm{~T} 4-8$ & 0.17 & 0 & 0 \\
\hline 2 & decreased & improved & $0.69(9: 13)$ & $\mathrm{T}-3$ & $\mathrm{C} 4-\mathrm{T} 10$ & 0.48 & $0.27(3: 11)$ & 0.07 \\
\hline 3 & decreased & not obtained & $0.64(14: 22)$ & $\mathrm{T}-9$ & $\mathrm{~T} 4-11$ & 0.41 & $0.47(8: 17)$ & 0.22 \\
\hline 4 & normal/decreased & unchanged & $0.42(5: 12)$ & C5-6 & $\mathrm{C} 4-\mathrm{T} 2$ & 0.17 & $0.17(2: 12)$ & 0.03 \\
\hline 5 & not obtained & good flow & $0.70(14: 20)$ & $\mathrm{C}-3$ & $\mathrm{C} 1-\mathrm{T} 1$ & 0.49 & $0.29(4: 14)$ & 0.08 \\
\hline
\end{tabular}

* The ratios of the pre- and postoperative syrinx/spinal cord diameter, and the syrinx/spinal cord surface area at the level of greatest expansion (epicenter) are reported. The sagittal extent of the syrinx (syrinx), as well as the results of the pre- and postoperative flow studies (preop flow, postop flow) are also noted.

and 4) who underwent both pre- and postoperative cine MR imaging demonstrated qualitative improvement of flow at the foramen magnum postsurgery. However, in a prospective reading of the preoperative studies obtained in Case 1 we found that the flow was normal. On retrospective comparisons of these studies to those obtained postoperatively, we suspected that flow at the craniocervical junction was compromised before surgery.

\section{Surgical Findings}

Intraoperative findings (Table 1) included the following: one patient (Case 1) had midline bone defects of the posterior arches of C-1 and C-2; another (Case 5) had assimilation of the posterior arch of $\mathrm{C}-1$ on the right; two (Cases 3 and 5) had significant intradural adhesions that were lysed; one (Case 2) had an obvious fourth ventricular "veil" of tissue that covered the opening of the central canal at the obex from the subarachnoid space. On disruption of the veil, good CSF flow was seen to emanate from the foramen of Magendie. In this same patient, the cerebellar tonsils were crowding the rostral aspect of the foramen magnum enough that we thought it would be helpful to shrink one tonsil with electrocautery. Finally, during operative exploration of the craniocervical junction in another patient (Case 3), the tonsils were noted to protrude mildly below the foramen magnum, although both prospective and retrospective evaluations of the preoperative MR images did not confirm that observation.

\section{Previous Surgery}

Prior to the craniocervical decompressions, two patients had undergone a syrinx-to-subarachnoid shunt placement. One of these (Case 2) had temporary improvement in syrinx size and scoliosis, both of which recurred within a few months. The second patient (Case 5) underwent two such shunt operations before the craniocervical decompression procedure. The first surgery produced only temporary improvement in hand weakness and syrinx size, whereas the second resulted no improvement in either. One patient was referred for evaluation after his brother, who had a typical Chiari I malformation, improved significantly after decompression.

\section{Postoperative Complications}

One patient experienced worsening of a cervicothoracic kyphosis several months postoperatively, and ultimately required a spinal fusion procedure. There were no compli- cations or surgical interventions in any of the other patients.

\section{DISCUSSION}

In this report, we describe five patients with presumably idiopathic syringohydromyelia who improved after a craniocervical decompression. The improvement was documented both clinically and radiographically, and there was a dramatic reduction in the size of the syrinx in all patients. Although the average follow-up period was relatively short (a little more than 1 year), the initial data are encouraging.

We infer from these results that, on occasion, the disturbance of CSF flow at the foramen magnum does not have to be associated with downward herniation of the cerebellar tonsils. Such a disturbance in fluid dynamics may be explained in one of our patients by the presence of a veil at the fourth ventricular outlet, and by arachnoidal adhesions in two other patients. In fact, within seconds of surgical lysis of these adhesions and veil, CSF flow from the fourth ventricle into the subarachnoid space seemed to improve. Of further importance is the observation that even though the tonsils were not located below the foramen magnum on the preoperative MR images, intraoperative exploration revealed crowding of posterior fossa structures at the level of the foramen magnum in two patients (Cases 2 and 3). Both of these patients underwent intradural decompression, and one of them underwent shrinking of the tonsil by means of electrocautery. These findings were coupled with evidence of flow compromise on preoperative cine MR imaging (Fig. 3), with improvement in flow postoperatively. However, although all four of the preoperative flow studies were found to be abnormal when evaluated retrospectively, the prospective readings of two of these studies did not document the abnormality. It was only when these studies were compared with the postoperative ones, which had all shown improvement, that the disturbance became suspect. However, of more significance than the patients with crowding of the posterior fossa contents are those with very adequate CSF ventral to the brainstem. These patients would seem to be the least likely to improve after posterior fossa decompression, yet they fared as well as the others.

In an older study of syringohydromyelia, Newton ${ }^{10}$ describes the presence of fourth ventricular drainage anomalies. In some cases, hindbrain herniation was also absent; however, because such cases were investigated in the 


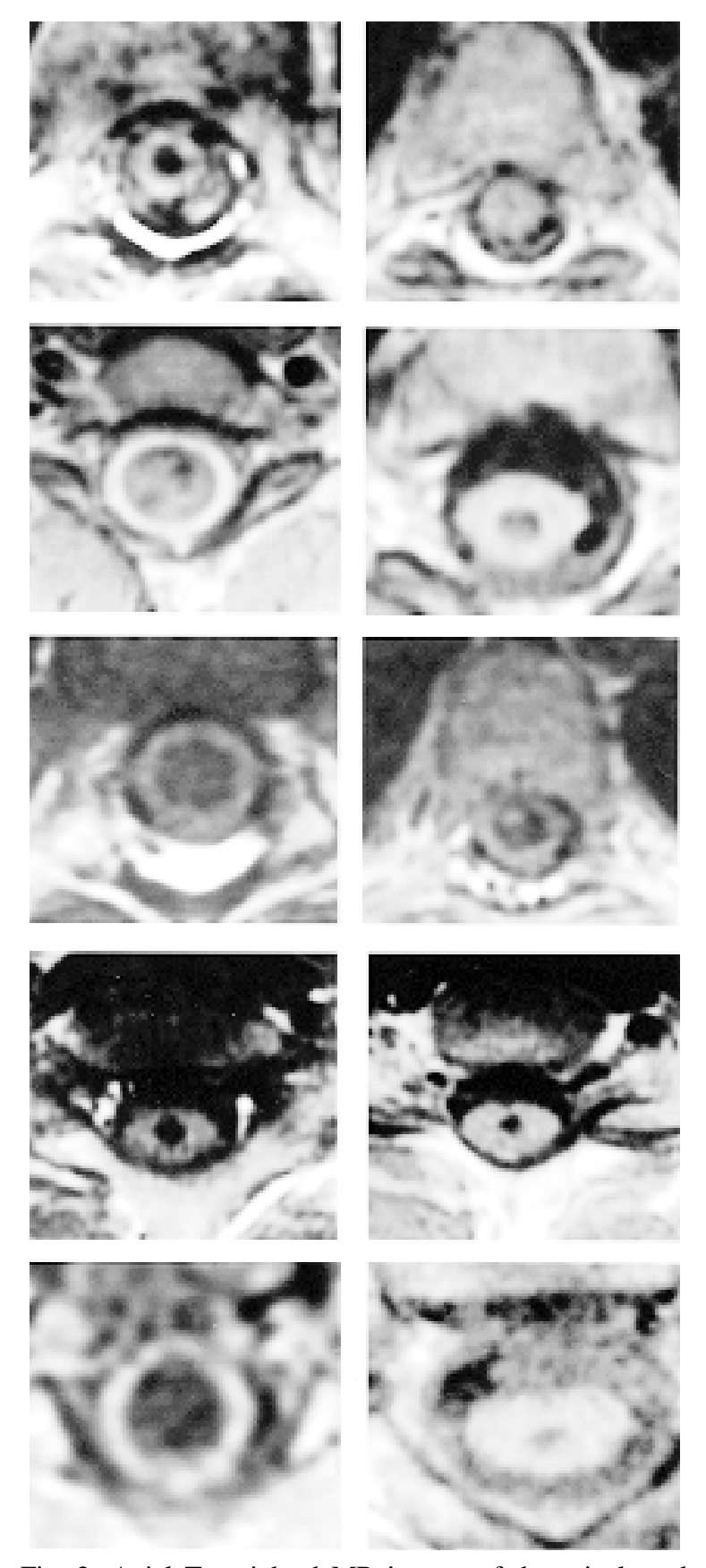

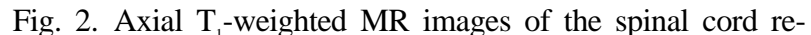
vealing the syrinx at the level of greatest preoperative expansion. The left column shows the preoperative images, and the right column shows the postoperative images at the corresponding spinal levels. These images represent Cases 1 to 5 , from top to bottom.

pre-CT/MR imaging era, radiological studies were limited to myelography and air ventriculography. Surgical treatment in these patients usually consisted of posterior fossa decompression in addition to syrinx drainage, with good results. In our series, draining the syrinx was deemed unnecessary.

The size (volume) of the posterior fossa is difficult to assess retrospectively because of the differences in slice

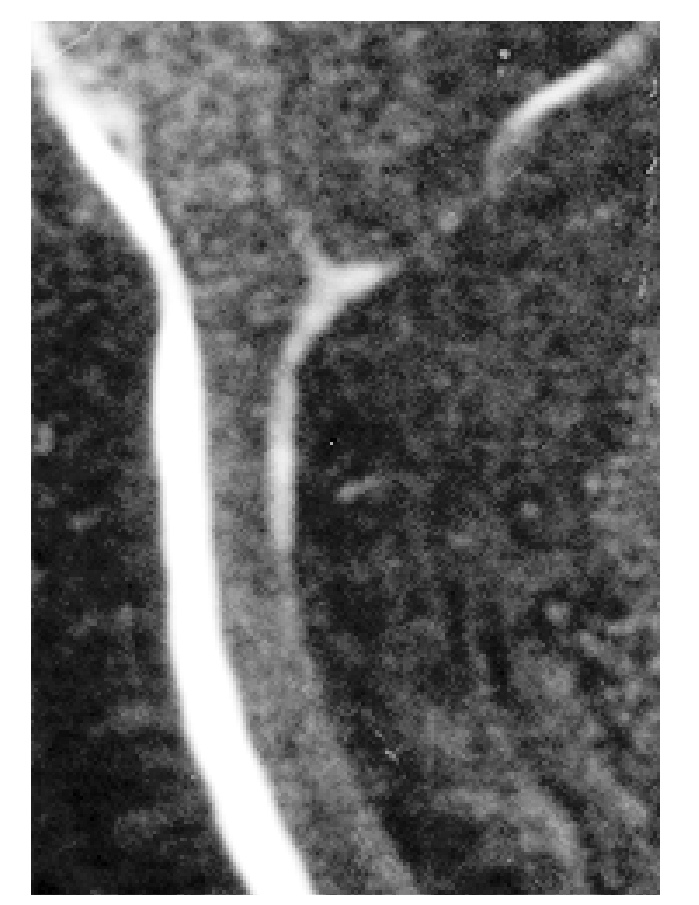

Fig. 3. Sagittal view of the craniocervical junction obtained by using the 2-D phase-contrast cine MR technique. Note the compromised CSF flow dorsal to the cerebellar tonsils.

acquisition on MR imaging among the patients, as well as between these patients and any standardized control group. A small posterior fossa could explain the phenomenon that we describe in some of the patients. However, that hypothesis cannot be confirmed with the available data. A prospective evaluation of such patients with standardized MR imaging protocols may help shed light on this issue in the future.

The use of cine MR imaging was not particularly helpful in this study. Although it has been attempted in some studies to compare the results of quantitative cine MR imaging among patients with normal brains and those with Chiari I malformations, ${ }^{2,4}$ we have not found any studies in which the use of this technique was standardized in a large control group. This makes our cine MR imaging data difficult to interpret. For instance, the improvement in flow noted after surgery in two cases may reflect the normal expansion of the subarachnoid space postoperatively.

Several pathogenetic theories have been proposed to explain the occurrence of Chiari malformations and the development of syringohydromyelia. Williams ${ }^{14}$ modification of Gardner's theory ${ }^{8,12}$ is probably the most appropriate when attempting to explain the findings in this study. Williams suggested that normally occurring valsalva maneuvers (coughing, straining, and sneezing) resulted in epidural venous congestion and intracranial as well as intraspinal pressure rises, causing fluid to flow both cranially and caudally. In the presence of craniocervical abnormalities, flow into the cranial compartment meets no resistance, whereas caudal flow may be delayed by hindbrain adhesions and outlet obstruction, thus creating a 


\section{Resolution of syringohydromyelia after posterior fossa decompression}

pressure differential between the cranial and spinal compartments. This pressure differential may last from a few seconds to several minutes and cause worsening hindbrain impaction. When the fluid cannot exit through the fourth ventricular outlet foramina because of this impaction or blockage, it may be rerouted into the central canal of the spinal cord, causing syringohydromyelia. In an attempt to give more credence to his theory, Williams measured the pressures in the caudal and spinal compartments before and after craniocervical decompression, showing equilibration of the pressures in the two compartments, which, in turn, correlated with clinical improvement. In our series, we were unable to detect a patent central canal rostral to the syrinx on MR imaging. In addition, two patients (Cases 1 and 3) demonstrated long skip areas between the foramen magnum and the rostral aspect of the syrinx (Figs. 1 and 3). These observations cast some doubt on the applicability of Williams' theory in this group of patients, and present the same controversies seen with the Chiari Iassociated syringohydromyelia. ${ }^{12}$ Authors who have attempted to resolve such controversies include Ball and Dayan, ${ }^{3}$ Aboulker, ${ }^{1}$ and Pillay, et al. ${ }^{12}$ Ball and Dayan hypothesized that CSF under pressure may enter the spinal cord through Virchow-Robin spaces, and gave indirect evidence of their theory by demonstrating dilated vessels and perivascular spaces in spinal cord autopsy specimens. Albouker noted that a large amount of CSF is produced in the spinal cord. When associated with an obstruction at the level of the foramen magnum, such fluid accumulates, causing the formation of a syrinx. Finally, Pillay, et al., attempted to combine these hypotheses into one unified theory of syringohydromyelia. However, to this date no theory of formation of syringohydromyelia has accounted for all the features of such anomalies.

Although idiopathic cervical syringohydromyelia may respond to posterior fossa decompression, we caution that the presence of syringohydromyelia in patients with no Chiari I malformation is much more likely to be caused by serious diseases, such as spinal cord tumors. It is imperative that a thorough investigation for tumor, posttraumatic arachnoiditis, and spinal dysraphism be conducted prior to any operative intervention. Furthermore, we encourage surgeons to use confirmatory specialized studies such as cine MR imaging, which measure the flow of CSF at the craniocervical junction, keeping in mind that such studies have not yet been optimized and standardized in large numbers of normal patients.

\section{References}

1. Aboulker J: La syringomyelie et les liquides intra-rachidiens. Neurochirurgie 25 (Suppl 1):1-44, 1979
2. Armonda RA, Citrin CM, Foley KT, et al: Quantitative cinemode magnetic resonance imaging of Chiari I malformations: an analysis of cerebrospinal fluid dynamics. Neurosurgery 35: 214-224, 1994

3. Ball MJ, Dayan AD: Pathogenesis of syringomyelia. Lancet 2:799-801, 1972

4. Bhadelia RA, Bogdan AR, Wolpert SM, et al: Cerebrospinal fluid flow waveforms: analysis in patients with Chiari I malformation by means of gated phase-contrast MR imaging velocity measurements. Radiology 196: 195-202, 1995

5. Chiari H: Über Veränderungen des Kleinhirns, des Pons und der Medulla oblongata in Folge von congenitaler Hydrocephalie des Grosshirns. Denkschr Akad Wiss 63:71-116, 1895

6. Chiari H: Ueber Veränderungen des Kleinhirns infolge von Hydrocephalie des Grosshirns. Dtsch Med Wochenschr 17: 1172-1175, 1891

7. Enzmann DR, Pelc NJ: Normal flow patterns of intracranial and spinal cerebrospinal fluid defined with phase-contrast cine MR imaging. Radiology 178:467-474, 1991

8. Gardner WJ: Hydrodynamic mechanisms of syringomyelia: its relationship to myelocele. J Neurol Neurosurg Psychiatry 28:247-259, 1965

9. Levy LM, Di Chiro G: MR phase imaging and cerebrospinal fluid flow in the head and spine. Neuroradiology 32:399-406, 1990

10. Newton EJ: Syringomyelia as a manifestation of defective fourth ventricular drainage. Ann Roy Coll Surg Eng 44:194213, 1969

11. Oakes WJ: Chiari malformations and syringohydromyelia in children, in Rengachary SS, Wilkins RH (eds): Neurosurgical Operative Atlas. Baltimore: Williams \& Wilkins, 1991, pp 5965

12. Pillay PK, Awad IA, Hahn JF: Gardner's hydrodynamic theory of syringomyelia revisited. Cleve Clin J Med 59:373-380, 1991

13. Pujol J, Roig C, Capdevila A, et al: Motion of the cerebellar tonsils in Chiari type I malformation studied by cine phase-contrast MRI. Neurology 45:1746-1753, 1995

14. Williams B: Cerebrospinal fluid pressure-gradients in spina bifida cystica, with special reference to the Arnold-Chiari malformation and aqueductal stenosis. Dev Med Child Neurol Suppl 35:138-150, 1975

Manuscript received August 4, 1997.

Accepted in final form March 24, 1998.

This manuscript was presented at the American Association of Neurological Surgeons/Congress of Neurological Surgeons Joint Section of Pediatric Neurosurgery, New Orleans, Louisiana in December 1997 under the title "The Chiari Zero Malformation."

This manuscript was previously published in the Journal of Neurosurgery, J Neurosurg 89:212-216, 1998.

Address reprint requests to: Bermans J. Iskandar, M.D., Department of Neurological Surgery, University of Wisconsin Hospital and Clinics, 600 Highland Avenue, H4/334, Madison, Wisconsin 53792. email: iskandar@neuro-novell.neurosurg.wisc.edu. 